\title{
Researching Technology and Equipment about the Vertical Billet in the Intelligent Manufacturing Production Line
}

\author{
Bo Zhong $^{1 *}$, Wenjin Zhou ${ }^{1}$, Yanlong Wang ${ }^{1}$, Qiqi Zhang ${ }^{1}$ \\ ${ }^{1}$ China building materials inspection and Certification Group Shaanxi Co., Ltd. Xi'an 710116, Shaanxi
}

\begin{abstract}
Firstly, this text is introducing the traditional technology of vertical billet. Secondly, the text is describing technological process and equipment principle about first and second generation vertical billet machine. Thirdly, the text is illustrating the second generation machine's superiorities by contrasting. Finally, we have put forward the thought of optimal to the second generation machine.
\end{abstract}

\section{Traditional technology}

In the traditional process, the mold is turned over manually after grouting, grouting, mold opening and drying. At this point, the blank body is completely separated from the mold and placed on the mold tray with the mold holder. After a certain period of natural drying, the blank body placed on the mold tray is transferred to the green plate by manual labor. Then the green slab with the green body is sent to the green body drying warehouse to realize the overall drying of the green body.

In order to fit the automatic production line, the original material of the mould tray is gypsum mould tray, the material is first changed to polystyrene film plastic, and the mould tray is connected with the pallet through adhesive, which ensures the overall portability of the pallet.

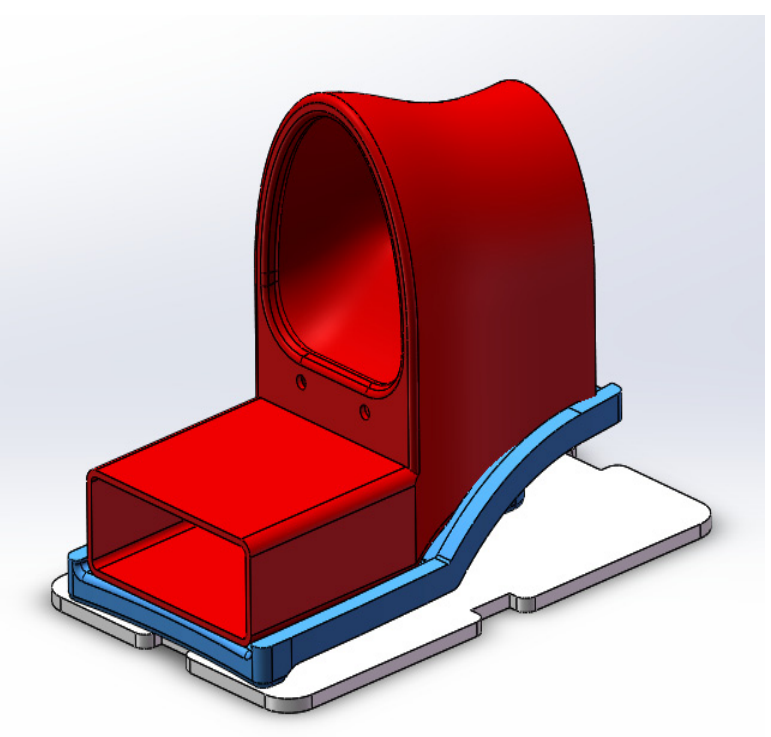

Fig 1. The position of the blank body on the die tray

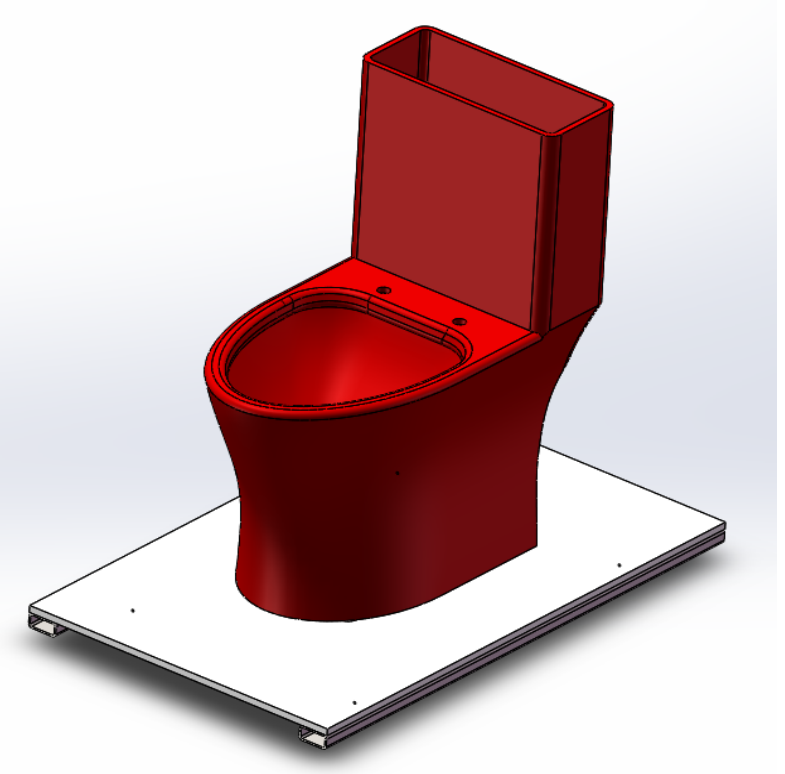

Fig 2. The position of a green body on a green plate

\section{First generation technological process of Vertical Billet}

The whole steel frame of the first generation billet standing machine is welded steel frame, and the left and right sides are equipped with auxiliary steel frame with oil pressure buffer, which is used to ensure that the rotating $\mathrm{L}$ arm can get good buffering and support in the initial position and the completion of $90^{\circ}$ rotation, so as to ensure the integrity of the billet body. The green plate tray stand and the die tray stand are installed at an Angle of $90^{\circ}$ on the rotating $\mathrm{L}$ arm to ensure that the entire rotation process is $90^{\circ}$ to prevent the green body from entrainment. The green plate tray stand and the die tray stand are attached with the top plate chain for transmission, and the chain drive is inside to ensure the whole transmission process is stable. The main rotating power mechanism is composed of $0.75 \mathrm{KW}$ ordinary motor and $\mathrm{I}=400$ helical gear worm 
reducer, which has high self-locking safety performance.

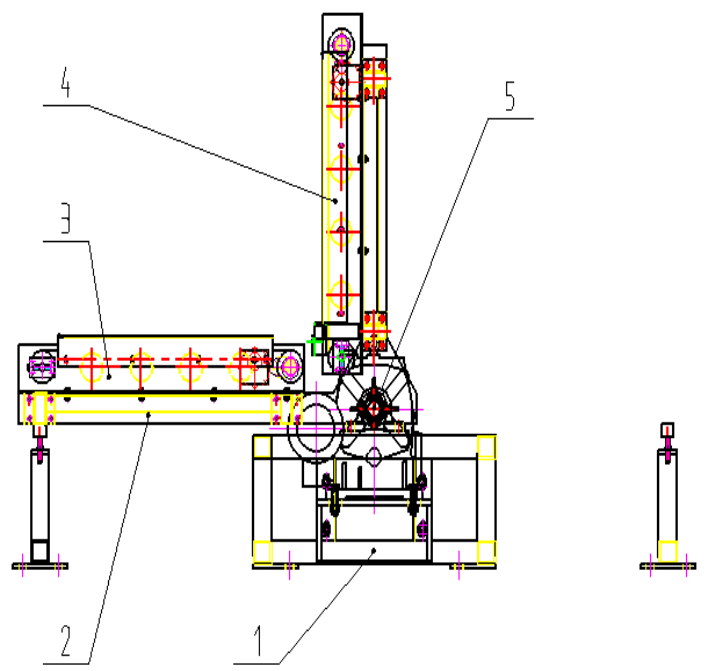

1. Monolithic steel frame 2. Rotating L arm 3. Green plate pallet rack 4. Rotating main power mechanism

Fig 3. Structure drawing of the first generation billet maker

Due to the existence of slab replacement in the billet vertical process, the left and right sides of the billet vertical machine should be equipped with a return machine to ensure the supply of green slab and the return plate of the die support plate. The specific technological process is as follows:

a) At the initial position, the empty green slab flows into the vertical billet from the left side of the vertical billet.

b) At this point, the green plate tray shelf starts to start, and the plate chain starts to run (the drive plate chain deceleration motor is $0.37 \mathrm{KW}, 1350 \mathrm{r} / \mathrm{min}, \mathrm{I}=60$, the plate chain sprocket is 17 tooth $08 \mathrm{~B}$ double row chain, the indexing circle diameter is $69.13 \mathrm{~mm}$ ). The running time is about 14 seconds before it reaches the specified position.

c) The rotating power mechanism starts and drives the rotating $\mathrm{L}$ arm to rotate clockwise $90^{\circ}$, which takes about 13 seconds.

d) After that, the die support plate carrying the billet body begins to flow into the billet machine from the right side of the billet machine.

e) At this point, the die tray stand starts and the plate chain starts to run (the drive plate chain system is the same as the green plate tray stand). The running time is about 14 seconds before the specified position is reached.

f) The rotating power mechanism starts to drive the rotating $\mathrm{L}$ arm to rotate counterclockwise $90^{\circ}$, and the rotation time is about 13 seconds.

g) After the pallet rack starts to run and the chain starts to run for about 14 seconds, the green plate loaded with the green body flows out of the billet vertical machine. h) The rotating power mechanism starts again and drives the rotating $\mathrm{L}$ arm to rotate clockwise by $90^{\circ}$. The rotation time is about 13 seconds.

i) After the die tray stand is started and the plate chain begins to run for about 14 seconds, the empty green slab will flow out of the billet forming machine.

j) The rotating power mechanism starts again and drives the rotating $\mathrm{L}$ arm to rotate clockwise by $90^{\circ}$. The rotation time is about 13 seconds. And then we go back to where we started.

Starting from the whole process, a blank cycle needs at least 1 minute and 40 seconds, which is a long time. If the production of the billet is too large, it is very easy to cause the pile-up on the mould tray in front of the vertical billet machine.

Starting from the mechanical structure, the power of the whole mechanism is installed at the position of the rotating shaft, where the torque is large and the reducer is loaded. And are $90^{\circ}$ reciprocating movement, the reducer output end can not reach full turn, such a long-term extreme impact of the reducer service life. In the process of rotation, the body weight center of the billet is shifted, and the return clearance of the worm gear reducer is large. Therefore, when the vertical billet machine is rotated to $80^{\circ}$, the torque direction of the output end of the reducer is instantly reversed, which will produce a large vibration and easily cause damage to the billet body.

Based on the above reasons against the billet machine optimization, research and development of the second generation billet machine.

\section{Second generation technological process of Vertical Billet}

The peripheral steel frame of the second generation billet standing machine is built of H-beam steel, with $180^{\circ}$ circular rack (modulus 8 , tooth number 186) hoisted on the upper part, and a supporting steel frame in the middle, which is used to install the rotating shaft, two side supports, support green billet steel frame and die bracket steel frame. The green slab strap frame and the die supporting slab strap frame are respectively mounted on the green slab steel frame and the belt steel frame. The internal drive system is a deceleration motor of $0.37 \mathrm{KW}, 1350 \mathrm{r} / \mathrm{min}, \mathrm{I}$ $=60$, and the outer diameter of the belt pulley is $130 \mathrm{~mm}$. The helical gear reducer with $0.75 \mathrm{KW}, 1400 \mathrm{r} / \mathrm{min}$, $\mathrm{I}=34.02$ and power lock is equipped in the green power system and the die support power system. It forms a soft connection with the green steel frame and the die support steel frame respectively through the unilateral directional wheel, and then crawls and clamps on the circular rack by relying on the gear (module 8 , tooth number 23 ) and two tensioning wheels. 


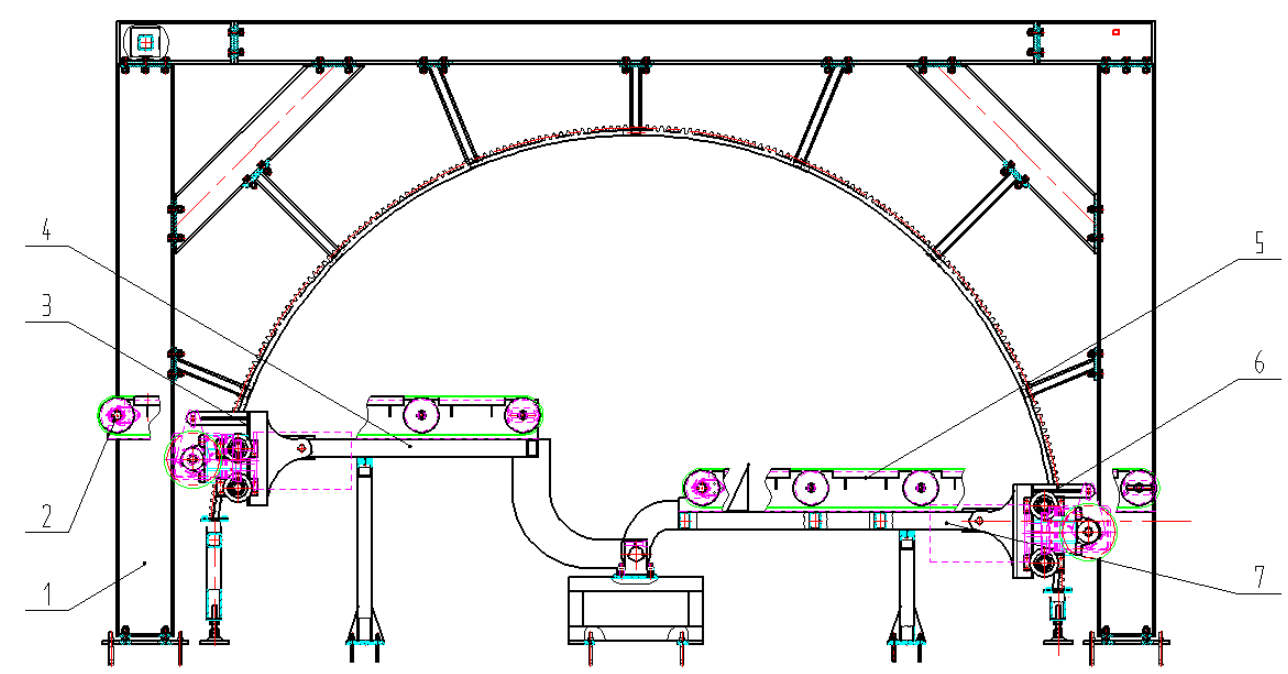

1. Peripheral steel frame 2. Green slab leather belt frame 3. Green power system 4. Green steel frame 5. Die pallet and strap frame 6. Module supporting power system 7. Die support steel frame

Fig 4. Structure drawing of the second generation billet maker

The precise positioning of the two kinds of tooling plates should be satisfied because the positioning of the bottom of the blank body is depended on when the blank is set up. Therefore, the green slab should be placed above the die tray before the billet standing. The specific technological process is as follows:

a) At the initial position, the left side of the empty green slab free-standing machine flows in, while at the same time, the right side of the free-standing machine of the die supporting plate carrying the blank flows in.

b) At this point, the green slab strap frame and the die tray strap frame start together. After running for about 9 seconds, the green slab and the die tray arrive at the designated position in turn. Because the green plate needs to be above the die plate when the blank is set up, the die plate is the first to reach the specified position compared with the green plate.

c) The green power system starts clockwise, drives the green steel frame, and relies on the gear to crawl on the circular rack of the peripheral steel frame, thus achieving the purpose of $90^{\circ}$ turning over. After about 8 seconds the two plates are perpendicular.

d) After that, the green plate power system and the die support power system start counterclockwise together, and the speed of both should be the same, so as to ensure the Angle of $90^{\circ}$ between the green plate and the die support. Complete $90^{\circ}$ rotation after approximately 8 seconds.

e) Then, the green slab strap frame reverses and starts clockwise with the mould supporting power system. After 8 seconds, the green slab carrying the green slab flows out of the vertical machine from the left side, and the mould supporting power system also makes the mould supporting plate strap frame reach the horizontal position.

f) Finally, the die pallet and belt stand is reversed and started. About 8 seconds later, the right side of the die pallet free-standing machine flows out of the billet standing machine. At this time, the billet standing machine completes a cycle, and the stations on both sides return to the initial state.

Starting from the whole technological process, the whole billet standing process can reach as short as 41 seconds, which accelerates the speed of billet standing and improves the transport speed of the whole production link. The process flow consists of one closed loop in one generation and is optimized to two closed loops. Fundamentally solved the problem of long time of the beat.

Starting from the mechanical mechanism, the bevel gear reducer is used to effectively reduce the backhaul clearance and avoid the large vibration generated in the center offset position. The original roof chain is changed to a pulley with a large axle diameter, which increases the friction coefficient during transmission and ensures that the plate will not slip or deviate during transport. The transmission system relies on the deceleration ratio between the circular rack and the gear, which reduces the deceleration ratio of the transmission reducer from the source and ensures the service life of the reducer. Placing power outside the radius of rotation rather than at the center of the circle also reduces the load on the motor itself.

\section{Future Optimization Outlook}

The second generation billet vertical machine solves the important problems existing in the first generation billet vertical machine in terms of technology and mechanical structure. But the second generation billet vertical machine still has the deficiency. For example, pinion and rack transmission process wear, noise. If the large rack quenching treatment will undoubtedly increase the processing cost of the whole equipment. The personal suggestion is to change the gear material to nylon, which can not only guarantee the life of the big rack, but also the nylon material is relatively soft, which can effectively reduce the noise produced by the whole operation process.

\section{Acknowledgments}

This work was supported by the study was supported by Key Research and Development Program of Shaanxi Province, China (No.2018ZDCXL-GY-06-02) 


\section{References}

1. Li Meichuan, Zhang Qi. Design and Research of Intelligent Manufacturing Line for Sanitary Ceramic[J]. China Building Materials Science \& Technology,2018:71-75

2. Zhang Qi, Li Meichuan. Technology and Design of Intelligent Casting and Grouting Circulation Line for Sanitary Ceramics $[\mathrm{J}]$. China Building Materials Science \& Technology,2018:82-84

3. Wu Xie, Chen Zhenhua, Li Fuying. Preparation of alumina ceramic elbow by grouting method [J]. China ceramics, 2011,47 (08): 50-53

4. Deng Shichao. Research on digital design and manufacturing technology of toilet products [D]. Hunan University, 2011

5. Zhou Jun, pan yubai, Li Jiang, Zhang Wenxin, Kou Huamin, Liu Wenbin, Guo Jingkun. Preparation of YAG Transparent Ceramics by grouting with anhydrous ethanol $[\mathrm{J}]$. Acta inorganic materials, 2011,26 (03): 254-256

6. Wang Xingang, Liu Jixuan, Jian Yanmei, Zhang Guojun, Wang Peiling, ZrB_ Injection molding and pressureless sintering of $2-\overline{S i C}$ ceramics $[\mathrm{J}]$. Acta inorganic materials, 2009,24 (04): 831-835

7. Hao Hongshun, $\mathrm{Xu}$ Lihua, Zhen Xiaomeng, Yang Jianying, Liu Ming, Yin hang. Preparation of high wear-resistant alumina ceramics by slip casting process [J]. Acta silicate Sinica, 2008 (11): 1615-1619

8. Wang Wenli, Kang Yongfeng. The important role of process in the design and operation control of intelligent manufacturing production line [J]. Aviation manufacturing technology, 2016 (16): 48-51 $+62$

9. Gao Zhiquan. Dongguan strives to build more than 100 universal intelligent manufacturing production lines [n]. Dongguan daily, July 23, 2016 (A02)

10. Bai Ruifeng, Han Honghong, Yu Heyang, Fang Zhaohui. Design and integration of virtual experimental system for intelligent manufacturing $[\mathrm{J}]$. Experimental technology and management, 2016,33 (06): $129-131+149$ 